\title{
An Adaptive Resource Allocation Scheme for Improving VoIP Capacity in Mobile WiMAX Systems
}

\author{
Soonuk Seol ${ }^{1}$ and Sungsoo $\mathrm{Cho}^{2}$ \\ ${ }^{1}$ Korea University of Technology and Education, \\ ${ }^{2}$ Advanced Institute of Technology, KT \\ suseol@koreatech.ac.kr,nicecho@kt.com
}

\begin{abstract}
In WiMAX networks, a base station (BS) sends DL-MAP and UL-MAP messages through downlink channel to represent transmission information, such as the size of slots/symbols and modulation/coding scheme (MCS), etc., for each of downlink and uplink data bursts. However, the MAP messages act as overheads. Particularly, in a service flow (SF) with the unsolicited grant service (UGS) schedule type, it is inefficient to allocate radio resources for transmitting the MAP messages on every grant interval because the grant interval and the requested bandwidth are fixed in UGS. In this paper, we propose an adaptive scheme using policy and charging control (PCC) architecture to reduce the MAP overheads in WiMAX networks, and prove the network capacity enhancements in the view of VoIP services.
\end{abstract}

Keywords: WiMAX, MAP, Overhead, Reduction, VoIP

\section{Introduction}

WiMAX networks refers to the standard IEEE 802.16 specifications [3] as MAC/PHY layer protocols. Figure 1 shows a WiMAX MAC frame structure. The MAC frame consists of downlink (DL) subframe and uplink (UL) subframe. A base station (BS) sends DL-MAP and UL-MAP messages through DL subframe to represent transmission information for each of downlink and uplink data bursts. This transmission information includes the size of slots or symbols, and modulation and coding scheme (MCS) and is specified by DL-MAP-IE or ULMAP-IE (Information Element) contained in DL-MAP or UL-MAP messages. For examples, if the BS is going to send a DL data burst (burst 1), then it sends transformation information (DL-MAP-IE) for the downlink data burst 1, and another DL-MAP-IE for the downlink data burst 2. Similarly, it is applied to uplink data burst transmissions. 


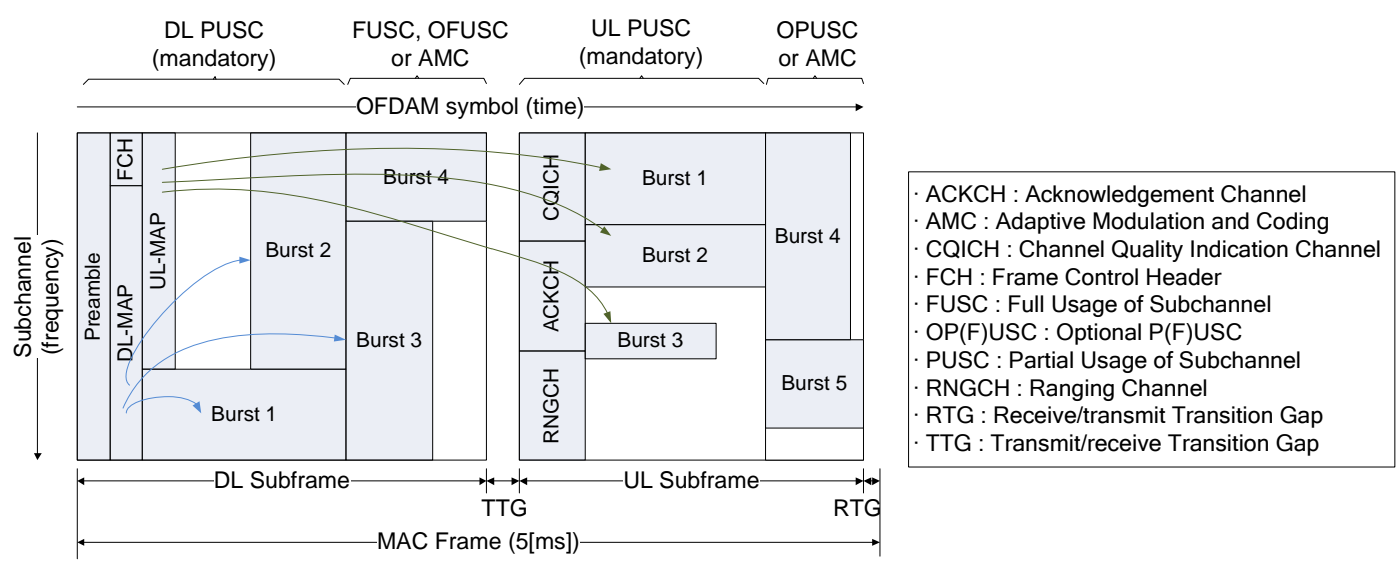

Figure 1. WiMAX MAC frame

Since a mobile station (MS) uses these MAP messages for decoding downlink data bursts and encoding uplink ones, the MAP messages are essential in the WiMAX networks. However, the MAP messages also occupy radio resources, and thus act as overheads. Specifically, although a UGS SF [4] to guarantee a certain of network bandwidth does not need the overheads for bandwidth allocation, it needs UL-MAP message for uplink data transmission, still. However, because the grant interval and requested bandwidth are already known as constants in UGS SF, it must be inefficient usages of radio resources to send MAP messages on every grant interval [2]. The previous study [1] had tried to reduce the MAP overheads, but, since it assumes persistent resource allocations, it is not adaptive to variable radio environments. In this paper, we propose an adaptive scheme using policy and charging control (PCC) architecture to reduce the MAP overheads in WiMAX networks, and prove the network capacity enhancements in the view of VoIP services.

\section{Adaptive MAP Reduction Scheme}

In this section, we explain our proposed scheme. Figure 2 represents the $\mathrm{PCC}$ architecture in WiMAX networks [4]. In this architecture, policy and charging rule function (PCRF) receives service information from application function (AF), such as proxy call session control function (P-CSCF) in the IP multimedia subsystem (IMS), through Rx interface. Then, it determines the quality of service (QoS) and charging policies, and manipulates the policies on units of service flow through Gx interface. Policy and charging enforcement function (PCEF) generally contained in access service network gateway (ASN-GW) enforces the policies received from the PCRF. This architecture is also specified in 3GPP PCC [5-7]. 


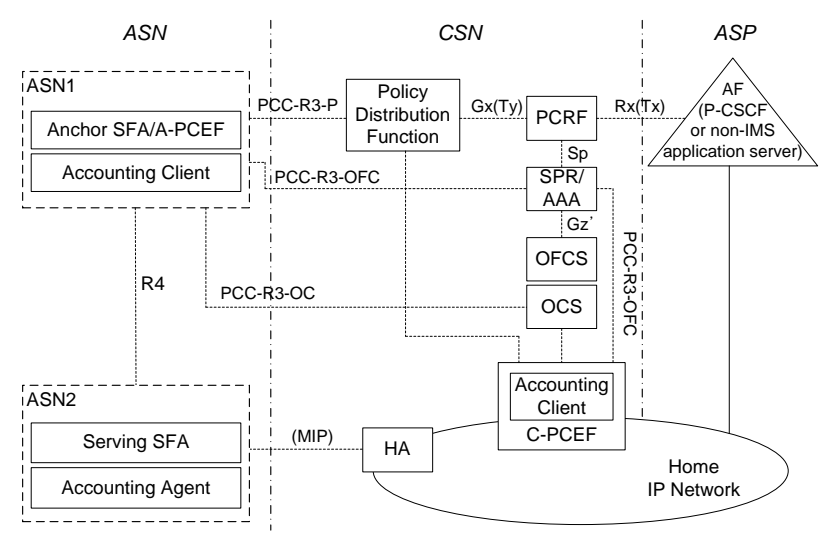

Figure 2. WiMAX PCC architecture

Figure 3(a) describes the WiMAX PCC signaling procedure to establish a dynamic service flow (DSF), of which uplink schedule type is UGS, for a MS triggered by an AF [4]. Initially, an AF may trigger the procedure. It requests the PCRF to establish a QoS policy for specific service information. The AAR message contains service information such as framed-IP, flow description (5-tuple), service bandwidth, and so on. Then, the PCRF decides the QoS policy including schedule type, requested bandwidth, grant interval, and so on, considering network topologies and environments. Then, the PCRF sends a RAR message to PCEF. The RAR message is shown in Figure 3(b). It contains various QoS parameters such as schedule type, unsolicited grant interval, bandwidth, latency, and so on for the IP flow specified by the flow description in the above AAR message. According to the QoS parameters in the RAR message, the PCEF adds a service flow for the designated MS. As explained above, the conventional WiMAX PCC architecture provides QoS for each service flow.

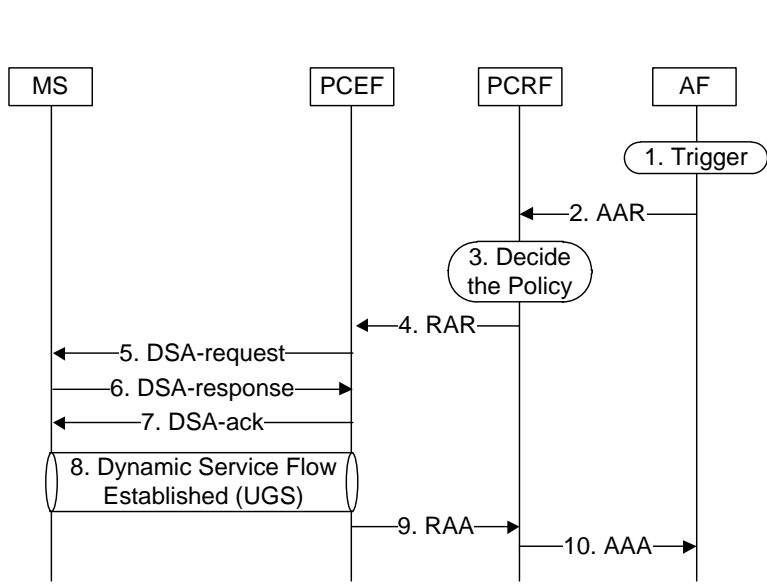

(a) DSF establishment procedure

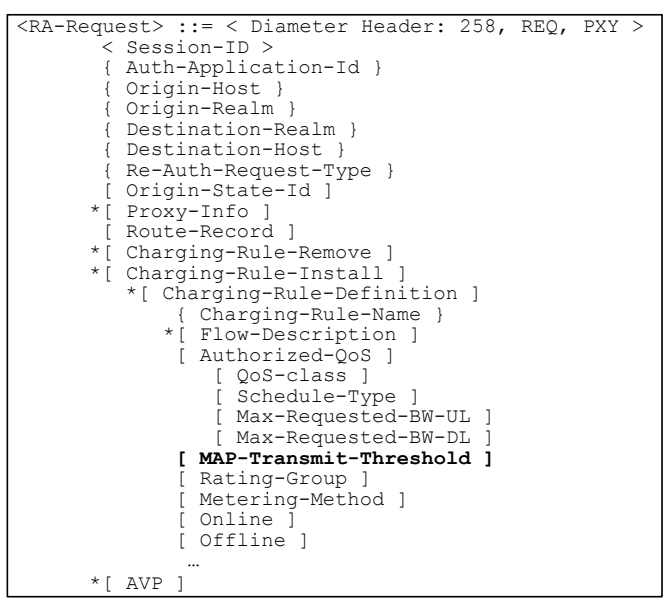

(b) RAR message with $M_{\text {th }}$ parameter

Figure 3. UGS DSF establishment

In our proposed scheme, the RAR message has been modified by defining a new additional parameter, MAP-Transmit-Threshold, the threshold $\left(\mathrm{M}_{\mathrm{th}}\right)$ of the number of MAP messages can be repeated, which is a natural number. Using the UGS SF specified with the proposed parameter $\mathrm{M}_{\mathrm{th}}$, we can design mechanisms to reduce MAP overheads between a BS and a MS. 


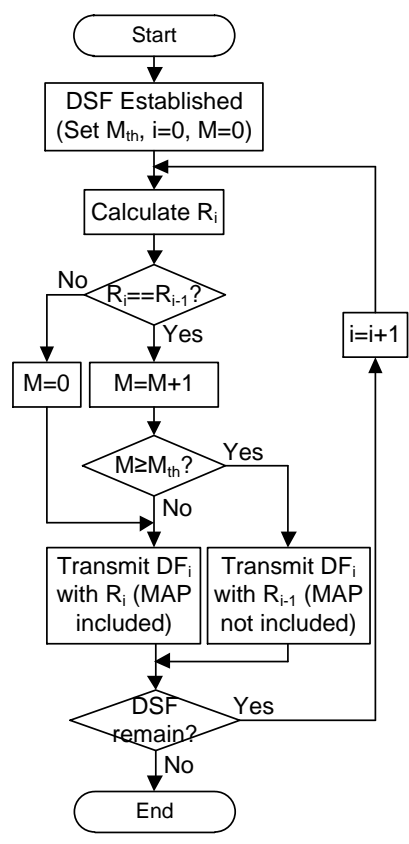

(a) BS

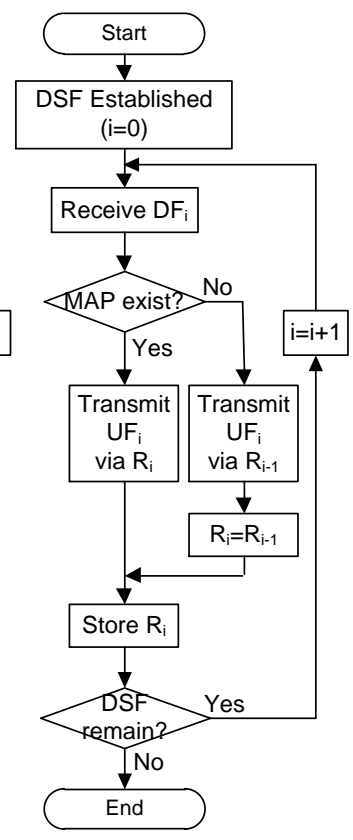

(b) MS

Figure 4. Adaptive UL-MAP-IE reduction mechanism

Figure 4 shows procedures to reduce UL-MAP-IE overheads which are used to designate transmission information of uplink data bursts on a DSF. As depicted in Figure 4(a), the BS allocates transformation information $\left(\mathrm{R}_{\mathrm{i}}\right)$ for an uplink data burst in i-th MAC frame. It is determined by various factors such as the size of a data burst, channel quality indicator (CQI), and so on. If $R_{i}$ is the same with the previous one $\left(R_{i-1}\right)$, the $B S$ increases a counter $M$ by 1 . Otherwise, it resets the counter to $0(\mathrm{M}=0)$. Then, the $\mathrm{BS}$ compares $\mathrm{M}$ with $\mathrm{M}_{\mathrm{th}}$. If $\mathrm{M}$ is equal to or greater than $\mathrm{M}_{\mathrm{th}}$, the BS does not transmit the UL-MAP-IE in the $\mathrm{i}$-th downlink frame $\left(\mathrm{DF}_{\mathrm{i}}\right)$. This procedure is repeated on every MAC frame with increasing the index i by 1. Figure 4(b) illustrates behavior of the MS. When the MS receives the $\mathrm{DF}_{\mathrm{i}}$, it confirms whether there is the UL-MAP-IE. If it does not exist, the MS transmits an uplink data burst in the $\mathrm{i}$-th uplink frame $\left(\mathrm{UF}_{\mathrm{i}}\right)$ based on the previous transmission information $R_{i-1}$. Then, the MS maps the $R_{i-1}$ to $R_{i}$ and stores it in its own memory because it may be used for the next frame $\left(\mathrm{UF}_{\mathrm{i}+1}\right)$. However, if the UL-MAP-IE exists in the $\mathrm{DF}_{\mathrm{i}}$, the MS transmits the $\mathrm{UF}_{\mathrm{i}}$ according to the $\mathrm{R}_{\mathrm{i}}$ designated by the UL-MAP-IE. In this case, the MS also stores $\mathrm{R}_{\mathrm{i}}$ in its own memory for $\mathrm{UF}_{\mathrm{i}+1}$. This procedure is repeated on every MAC frame until the SF is terminated. In this way, we can reduce the ULMAP-IE overheads, and thus save the radio resources of the downlink MAC frame. Likewise, we can also apply the proposed scheme to the DL-MAP-IE.

\section{Performance Evaluation}

We evaluate the downlink cell capacities of VoIP services in the WIBRO network deployed in Korea based on IEEE 802.16 [3] and WiMAX specifications [4]. The analysis environments are summarized in Table 1. As explained in Figure 1, WiMAX MAC frame, specifically DL subframe, has 27 time symbols totally. Among them, various overheads such as preamble, FCH and MAP messages occupy the resources. The overheads are divided into fixed and variable. Particularly, the DL-MAP-IE or UL-MAP-IE messages are variable because they are generated for each data burst. Then, available resources in DL subframe can 
be calculated as $24.542-\left(\mathrm{S}_{\mathrm{DL}-\mathrm{MAP}-\mathrm{IE}} * \mathrm{~N}_{\mathrm{DL}}+\mathrm{S}_{\mathrm{UL}-\mathrm{MAP} \text {-IE }} * \mathrm{~N}_{\mathrm{UL}}\right)$. Then, the downlink cell capacity of VoIP services is determined by the amount of the available resources. Meanwhile, we assume that modulation and coding scheme (MCS) is "QPSK, 1/2, 6 [repetition]" for MAP messages and "16QAM, 3/4" for data bursts, but "QPSK, 1/2" in the case of the persistent MAP reduction scheme. Also, the VoIP codec specifications are also listed in Table 1. In this analysis, we assumed five codecs used widely in VoIP services. Finally, in our proposed scheme, we let the probability of the number of ' $R_{i}$ equals to $R_{i-1}$ ' events in a UGS SF be a Poisson random variable with a mean 0.4 , and $\mathrm{M}_{\mathrm{th}}=1$.

Table 1. Environments for analysis

\begin{tabular}{c|c|c}
\hline & Parameter & Value [unit: symbols] \\
\hline \multirow{4}{*}{$\begin{array}{c}\text { WIBRO downlink } \\
\text { system parameters }\end{array}$} & Total resources & 27 \\
\cline { 2 - 3 } & Fixed overhead & $\begin{array}{c}\mathrm{S}_{\text {preamble }}=1, \mathrm{~S}_{\mathrm{FCH}}=0.125, \\
\mathrm{~S}_{\text {DL-MAP-Fixed }}=0.8667, \\
\mathrm{~S}_{\text {UL-MAP-Fixed }}=0.4667\end{array}$ \\
\cline { 2 - 3 } & Variable overhead & $\begin{array}{c}\mathrm{S}_{\text {DL-MAP-IE }}=0.4334, \\
\mathrm{~S}_{\mathrm{UL}-\mathrm{MAP}-\mathrm{IE}}=0.2667\end{array}$ \\
\cline { 2 - 3 } & Usable resources & $\begin{array}{c}24.542-\left(\mathrm{S}_{\mathrm{DL}-\mathrm{MAP}-\mathrm{IE}} * \mathrm{~N}_{\mathrm{DL}}\right. \\
\left.+\mathrm{S}_{\mathrm{UL}-\mathrm{MAP}-\mathrm{IE}} * \mathrm{~N}_{\mathrm{UL}}\right)\end{array}$ \\
\hline \multirow{3}{*}{$\begin{array}{c}\text { Modulation and coding } \\
\text { scheme (MCS) }\end{array}$} & MAP message & QPSK, 1/2, 6[rep] \\
\cline { 2 - 3 } & DL data burst & $16 \mathrm{QAM}, 3 / 4$ \\
\cline { 2 - 3 } & DL data burst in persistent & QPSK, $1 / 2$ \\
\hline \multirow{4}{*}{ VoIP codec } & MAP reduction scheme & $8.6[\mathrm{kbps}], 20[\mathrm{~ms}]$ \\
\cline { 2 - 3 } & EVRC & $13[\mathrm{kbps}], 20[\mathrm{~ms}]$ \\
\cline { 2 - 3 } & GSM6.10 & $64[\mathrm{kbps}], 20[\mathrm{~ms}]$ \\
\cline { 2 - 3 } & G.711 & $5.3[\mathrm{kbps}], 20[\mathrm{~ms}]$ \\
\cline { 2 - 3 } & G.723.1 & $8[\mathrm{kbps}], 20[\mathrm{~ms}]$ \\
\hline
\end{tabular}

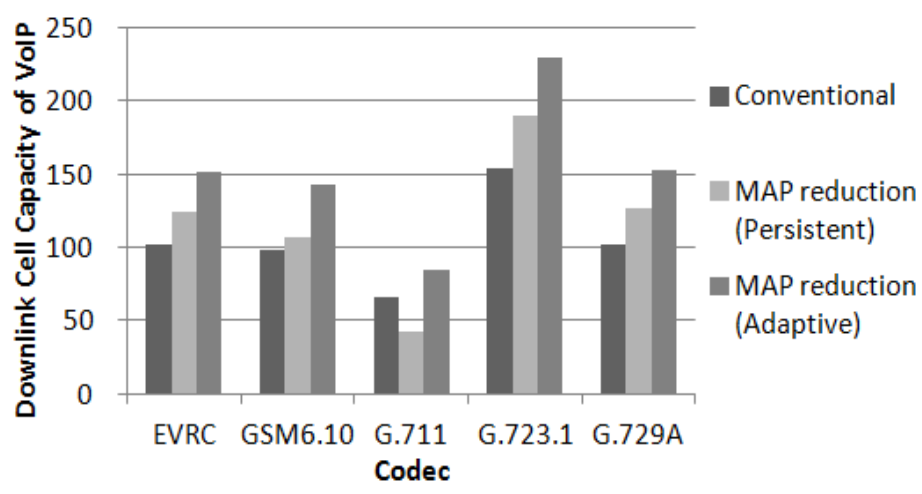

Figure 5. Downlink cell capacity of VolP services

Figure 5 illustrates the analysis results for five codecs. It is shown that our proposed scheme enhances downlink VoIP capacity by about 20 50 [\%] compared to the conventional one and the persistent MAP reduction schemes. In the conventional scheme, all data bursts entail unnecessary UL-MAP-IEs, resulting in inefficient network resource usages. In case of the persistent MAP reduction scheme, it does not use any MAP messages, but it uses constant MCS (QPSK, 1/2), which is very strong, to data bursts at any radio environments. Thus, it may induce inefficient radio resource usages even in good radio environment (high carrier-to- 
interference-and-noise ratio (CINR)). Contrarily, our proposed scheme does not transmit any UL-MAP-IE in order to save downlink radio resources when the event ' $R_{i}$ equals to $R_{i-1}$ ', occurs, and uses MCS adaptively with respect to the wireless environments. In other words, since our new scheme considers variations of radio channel environment and does not use MAP messages in consistent radio environment, it can enhance the efficiency of radio resource usages.

\section{Conclusion}

In this paper, we proposed an adaptive resource allocation scheme in WiMAX networks. We showed that the scheme enhances the network capacity, specifically, when UGS service flows are used where the same MAP messages are likely to be transmitted repeatedly. In further study, we will apply our proposed scheme to 3GPP Long-Term Evolution (LTE) networks.

\section{References}

[1] M. Fong and R. Novak, "Improved VoIP Capacity in Mobile WiMAX Systems Using Persistent Resource Allocation”, IEEE Communications Magazine, (2008) October.

[2] S. Seol, S. S. Cho and H. S. Kim, "Implementation of the standard WiMAX QoS framework and the verification of quality and capacity of mobile VoIP service", 5th proceeding, TTA, (2009), pp. 53-75.

[3] IEEE Standard 802.16e/D11-2005, "IEEE Standard for Local and Metropolitan Area Networks Part 16: Air Interface for Fixed Broadband Wireless Access Systems", (2005) September.

[4] WiMAX Rel 1.5, Draft-T33-109-R015v01-A(Working Group Approved Specification), “ WiMAX Forum Network Architecture - Policy and Charging Control”, (2008) December.

[5] 3GPP TS 29.214, release 9, "Policy and charging control over Rx reference point", (2013).

[6] 3GPP TS 29.213, release 9, "Policy and charging control signaling and Quality of Service (QoS) parameter mapping", (2013).

[7] 3GPP TS 29.212, release 9, "Policy and charging control over Gx reference point", (2013).
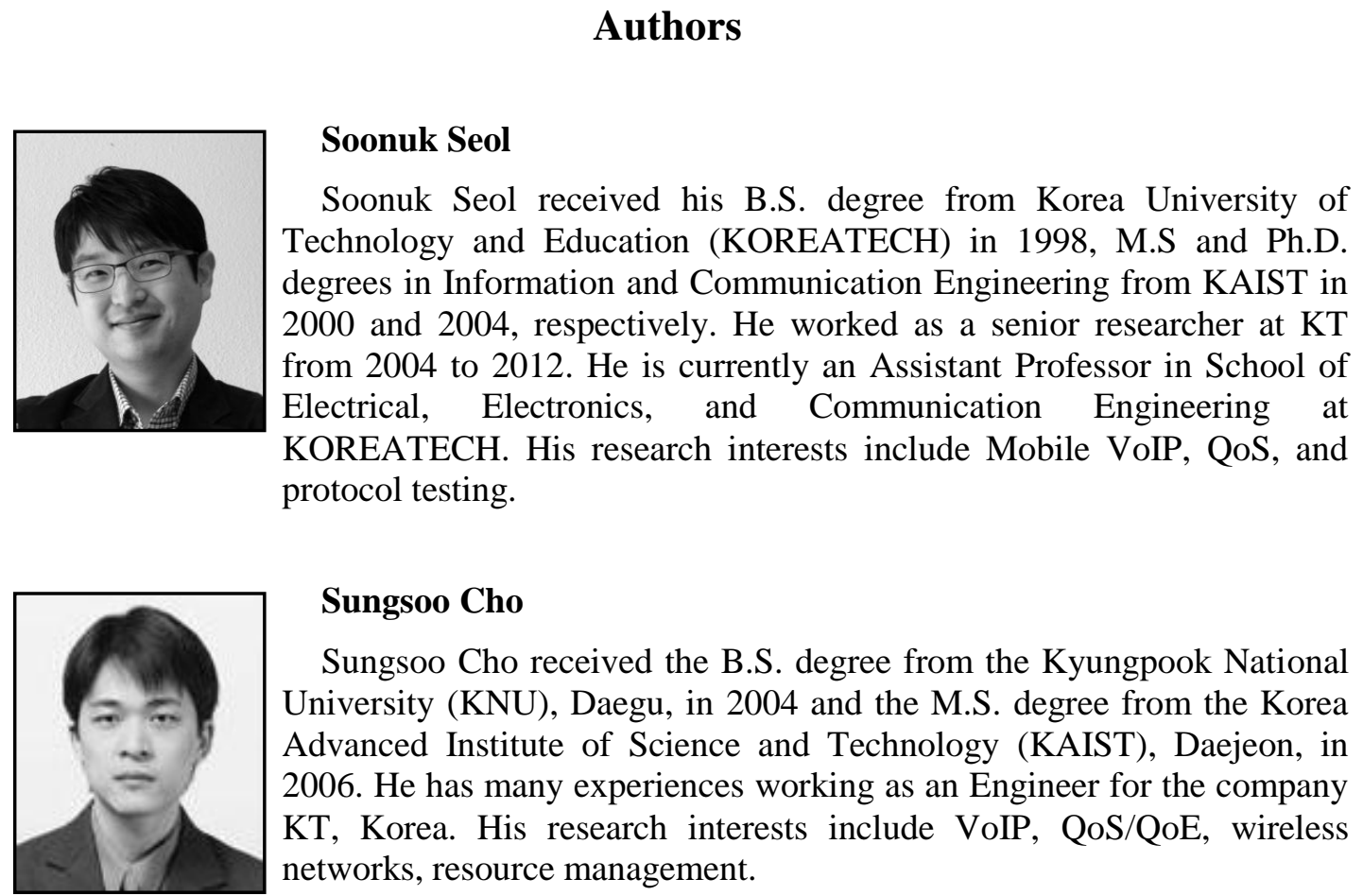

\section{Sungsoo Cho}

Sungsoo Cho received the B.S. degree from the Kyungpook National University (KNU), Daegu, in 2004 and the M.S. degree from the Korea Advanced Institute of Science and Technology (KAIST), Daejeon, in 2006. He has many experiences working as an Engineer for the company KT, Korea. His research interests include VoIP, QoS/QoE, wireless networks, resource management. 